also 8 metastases in entoderm organs-- the liver, the lungs and the pancreas-but the metastases in these instances were apparently not selective but due to causes previously discussed. It is also not unlikely that selection is greater in growths derived from more highly differentiated èntodermal glandular tissue.

Although the material analyzed is relatively: small a suggestion is contained in it that embyrogenetic and biological relationship of tumor tissue to physiological tissues may influence the often strangc selective metastases.: In other words it would appear as if closc embryogenetic affinity of tumor cells to tissues of the same derivation influence metastatic selection. It is well known that lymphosarcomata metastasize with preference in lymphadenoid, or mesodermal tissues.

The conclusion is therefore reached that the localization of metastases depends upon a number of factors. Important among thesc are:

1. The quantity of the tumor elements and the metbod of dissemination.

2. Effects of injurious metabolic products of tumor cells upon a tissue, causing degeneration and inflammation and thereby weakening its physiological resistance.

3. Close biogenetic (embryonic) relation of tumor cells to a tissuc soil, whereby types of tumor cells derived from an embryonic layer grow more readily in the environment of organs or tissues which are derived from the same layer of the blastoderm.

Accurate determination of this last point will only be possible with a large amount of tumor material analyzed grossly and microscopically with full regard to the factors discussed above.

\title{
ISOLATION OF THE MENINGOCOCCUS FROM CASES OF SO-CALLED INFLUENZA.
}

By Majon Roger KInnicutT, M.C., U.S.A.,

AND

Fingt Lieüt. Carl A. L. Binger, M.C., U.S.A.

INTRoDucrion. Material for the following bacteriological study was obtained from cases occurring in two epidemics of so-called influenza and from a number of scattered cases admitted to the U. S. Base Hospital No. 6, Bordeaux, France. The first epidemic spread among a forestry organization stationed in and about Mimizan in the Department of Landes. In the three camps affected, 
approximately 350 cases appeared in a strength of 553; about 30 of these were complicated by bronchopneumonia and 15 died. The condition encountered presented an almost constant. symptomatology with a few minor variations; headache and generally bodily pains of rather sudden onset, elevation of temperature lasting from three to ten days, relatively slow pulse-rate, considerable prostration and signs of $a$ diffuse bronchitis. Nose-bleed was a common $\cdots \cdots$ : feature. In - the fatal cases there was delirium, deep cyanosis and evidence of extensive bronchopneumonia consolidation.

Cases in the second epidemic investigated presented a similar clinical picture. At this time tbe disease was still apparently in its "pure" form, not confounded by the great variety of respiratory infections encountered among troops in France during the fall and winter months. Diagnosis was therefore relatively simple and the selection of cases suitable for study presented no special difficulties. This epidemic oceurred during September and the first fortnight of Octoben, 1918, at Camp Hunt, Le Courneau, in the Department of Gironde. The epidemic seemed to be divided into two separate outbreaks, probably originally from different sources. The first began about August 10; there were about 800 cases, 28 pneumonia and 7 deaths. The second was imported to the Artillery Camp by the arrival of 3500 troops from Brest. In the two outbreaks 3915 cases developed requiring hospitalization, with 275 pneumonia and 65 deaths.

The scattered cases studied at Base Hospital No. 6 came from a variety of casual organizations stationed in the Base Section and from soldiers evacuated from the Zone of the Advance. These were more difficult of diagnosis and selection. The disease had lost, to a certain extent; its type, and confusion, with early lobar pneumonia, bronchitis and coryza, was hard to avoid. This may perhaps, to some extent, explain the divergent findings in this group of cases.

Technic Followed in Bacteriological Investigat.ons. For blood cultures the best medium was found to be ascitic agar or coagulated blood media.slants in flat-sided Pasteur tubes, the tubes being about one-quarter filled with dextrose infusion broth ( 0.4 per cent. acid).

For all other cultures a coagulated sheeps' blood medium was used, the formula for which is: Defibrinated sheeps' blood, 2 or 3 parts; dextrose infusion broth ( 0.2 per cent. to phenolphthalein), 1 part. 'The mixture is well shaken; slanted, inspissated and sterilized by fractional sterilization in an Arnold sterilizer. This medium is chocolate-colored, furnishing an excellent background for examining colonies, and partially differentiates the various micrococci. Upon it the meningococcus grows well; as rather large, round, gray, shiny, flat colonies, with smooth edges. Pneumococci and many of the streptococcus group grow as brilliant green colonies, with green discoloration of the medium. Other streptococci show a marked wbitish ring around the colonies. The common hemolytic streptococcus produces no color change. 
Cultures were obtained from the nasopharynx, sputum and blood of patients with influenza alone and with bronchopneumonia complicating the disease. At autopsies of cases dying of bronchopneumonia cultures were made from the hearts' blood and pneumionic areas in the lungs. : (Numbers : given indicate cultures from individual cases.)

The sera used for agglutination reactions. with Gram-negative diplocoeci isolated in this investigation were Types: $A ; B$ and $C$ meningococcus serum (French classification) obtained from the Pasteur Institute and in a few cases a polyyalent Rockefeller Institute antimeningococcus serum. Each of the test sera was used in dilutions of 1 to 25 and 1 to 50 . A normal horse serum control, dilution 1 to 25, was always used in each test. The bacterial suspensions were made in normal salt solution from pure cultures of Gram-negative diplococci grown on coagulated sheeps' blood medium.. All agglutination reactions were macroscopic and carried out in a water-bath at $37^{\circ} \mathrm{C}$.

Mnmzan Epidemic. Ten blood cultures were taken in dextrose broth. All but one showed no growth. One, taken two hours before death, gave a pure culture of meningococcus Type C. At the autopsy of this case meningococcus was not recovered from the heart's blood or lungs.

From the lungs of 4 cases out of 10 dying of bronchopneumonin meningococci were recovered at autopsy. From one case meningococci were recovered from an acute suppurative otitis media. All strains recovered agglutinated with the Rockefeller polyvalent antimeningococcus serum and not with normal horse serum. Only two of these strains isolated were typed; one was Type $C$ and the other Type B. (All strains isolated in this epidemic except the one obtained from blood were lost.) Engrs.

Protocols. No. 78, R. L. H., Sgt. 1Cl., Co. "E," 4th Bṭ., 20th

Clinical Diagnosis. Influenza; bronchopneumonia.

Autopsy. Extensive bronchopneumonia of all lobes of both lungs. Lung Cultures. - Meningococci, Type C, recovered from the left upper and the left lower lobes.

No. 79, G. A. B., Co. "E," 4th Btn., 20th Engrs.

Infuenza; bronchopneumonia.

Autopsy. Extensive bronchopneumonia involving all lobes of both lungs. lung.

Lung Cultures. Meningococci, Type B, recovered from the right

No. 80, C. C. C., Pvt., Co. "E," 4th Btn., 20th Engrs.

Influenza; bronchopneumonia. Blood culture in dextrose brotls taken two hours before death, Type C, meningococci, in pure culture. Autopsy. Extensive bronchopneumonia involving all lobes of both lungs. 
Lung Cullures. Meningococci not recovered.

No. 81, H. N., Pvt., Co. "F,", 4th Btn.,' 20th Engrs.

Influenza; bronchopmeumonia.

Autopsy. Extensive bronchopneumonia involving all lobes of both lungs. Acute sippurative otitis media (right).

Lung Cultures. Meningococci not recovered. Culture from right middle ear. - Gram-negative diplococci; agglutinated by Rockefeller Institute polyvalent antimeningococcus serum and not by normal horse serum. (Not typed, culture lost:)

A. W. W.; Pvt.; Co. "F,' 4th Btn., 20th Engrs.

Influenza; bronchopneumonia:

Autopsy. Extensive bronchop neumonia involving all lobes of both lungs.

Lang"Culliures. Gram-negative diplococci, agglutinated by Rockefeller Institute antimeningoccccus serim'and not by normal horse serum, recovered from right lower lobe. (Not typed, culture lost.)

C. H., Pvt., Co. “E," 4th Btn., 20th Engrs.

Influenza; bronchopneumonia.

Autopsy. Extensive bronchopneumonin, involving all lobes of both lungs.

Inng Cultures. Gram-negative diplococei, agglutinated by Rockefeller Institute polyvalent antimcningococcus serum and not by normal horse serum, recovcred from right upper lobe. (Not typed, culture lost.)

Le Courneau Epipearic. Nasopharyngeal Cultures. Twentyfive cultures were taken. Of these 22 showed Gram-negative diplococci. From 14 of these 22 cultures Gram-negative diplococci were isolated in pure culture; 9 of these 14 strains were agglutinated by Bockefeller Institute polyvalent antimeningococcus servim and not by normal horse serum; 5 strains were not agglutinated by either serum. The type of only two of these positive strains was determined. Both were agglutinated by Type $\mathrm{C}$ serum and not by Types A, B or normal horse serum.

Sputum. Specimens from only 4 cases were cultured. All showed Gram-negative diplococci, and from 2 these mikrococci were isolated in pure culture, both of which were agglutinated by Type $C$, serum and not by Types A, B or normal horse serum. In 1 case at the onset of the infection, Type $\mathrm{C}$ meningococcus was isolated from the nasopharynx, and at the beginning of convalescence from the sputum. This patient had influenza, with no complicating pneumonia.

Blood Cullures. The first 25 blood cultures were taken in broth and were all negative for Gram-negative diplococei. The last 15 blood cultures were taken in Pasteur tubes containing solid, slanted media as well as broth. 'Two or three times'a day the mixture of blood and broth was shaken and run up over the solid medium slants. This was done because the meningococcus is essentially an 
aërobe. Of these 15 cultures 4 showed Gram-negative diplococci. Of these 4 strains 3 agglutinated with Type $C$ serum, not with Types A, B or normal horse serum, the fourth, as yet, will not agglutinate with any of the sera on band. No blood cultures positive for Gram-negative micrococci were obtained except in fatal cases complicated by bronchopneumonia.

Heart's Blood. Of 24 cultures taken, 4 showed Gram-negativc diplococci: -Agglutination reactions were done with 3 of these strains; 1 was not agglutinated by any of the sera used, 2 agglutinated with a Type $C$ serum, not with Types A, B or normal horse serum.

Lungs. Twenty-two cultures were taken; 12 showed Gramnegative diplococci, 5 in almost pure culture. Of these 12 strains 11 agglutinated with Type $C$ sera, not with Types A, B or normal horse serum, and 1 did not agglutinate with any serum used.

Profocols. No. 13, R. E. B., Pvt., 5th Bty., F. A. R. R.

Infuenza; bronchopneumonia.

September 27, 1918. Blood culture in dextrose broth; no growth.

September 30. Blood culture in Pasteur tube; Gram-negative diplococci in pure culture (culture lost.)

No. 14, H. E. L., Pvt., 5th Bty., F. A. R. R.

Influenza; bronchopneumonia.

September 20, 1918. Blood culture in dextrose broth; no growtl.

September 22. Died.

Autopsy. Bronchopneumonia involving the whole of the right lung except the apex; left lower and part of the left upper lobss. Marked edema of the brain.

Cullures. Heart's blood: Streptococci and Type C meningococei.

Lungs: Streptococci and Type $\mathrm{C}$ meningococci.

No. 18, H. S., Pvt., 5th Bty., F. A. R. R.

Influenza; bronchopneumonia.

September 20, 1918. Blood culture in dextrose broth negative for meningococci.

September 22. Died.

Autopsy. Broncbopneumonia involving the right, upper, middlc and left lower lobes.

Cultures. Lungs: Gram-negative diplococci recovered from the lower lobe of the left lung.

No. 21, H. B., 5th Bty, F. A. R. R.

Influenza. Blood culture in dextrose broth; negative for menilgococci.

September 22, 1918. Died.

Autopsy. Beginning empyema (left.) Extensive' bronchopneumonia involving all lobes of both lungs.

Cultures. ..Lungs: Gram-negative diplococci, not agglutinated by any antimeningococcus serum on band, isolated from right lower and the left upper lobes. 
No. 36, H. F., Cpl. Hqs. Co., F. A. R. R.

Influenza. September 21, 1918. September 25. Died.

Autopsy. Bronchopneumonia involving the right upper, middle and the lower lobes and the left lower lobe.

Cullures: Lungs: Type $\mathrm{C}$ meningococci.

No. 39, A. C. B., Pvt., 4th Bty., F. A. R. R.

Influenza. September 23.

September 30. Bronchopneumonia.

Blood culture in Pasteur tubes. Type $\mathrm{C}$ meningococci in pure culture.

October 2. Died. Cultures not obtaind at autopsy.

No. 54, E. M., Sgt., 2d Bty., F. A R. R.

Influenza; bronchopneumonia....

September, 1918. Died.

Aulopsy. Extensive bronchopneumonia involving all the lobes of both lungs.

Lung Cullure. Type $\mathrm{C}$ meningococci recovered from the left upper lobe.

No. D. S., Pvt., 5th Bty., F. A. R. R.

Infuenza; bronchopneumonia.

September 25, 1918. Died.

Autopsy. Extensive bronchopneumonia involving all the lobes of both lungs.

Cultures. Lungs: Type $\mathrm{C}$ meningococci recovered from the right lower and the left lower lobes.

No. 60, W. T. M., Pvt., 4th Bty., F. A. R. R.

Infuenza; bronchopneumonia.

September 25, 1918. Died.

Autopsy. Beginning acute fibrinopurulent pericarditis. Empyema (left). Extensive bronchopneumonia, involving all lobes of both lungs.

Cultures. Lungs: Type $\mathrm{C}$ meningococci recovered from left upper lobe.

No. 73, F. W. G., Pvt., 2d Bty., F. A. R. R.

Infuenza; bronehopneumonia.

September 27, 1918. Died.

Autopsy. Einpyema (left.) Gelatinous exudate over the right lung. Extensive bronchopneumonia involving all the lobes of both lungs.

Cultures. Lungs: Type $\mathrm{C}$ meningococci in almost pure culture recovered from the rigbt lower, left lower and right upper lobes.

No. 78, W. H. G., Pvt., 2d Bty., F. A. R. R.

Infuenza; bronchopneumonia.

Autopsy. Exténsive bronchopneumonia involving all lobes of both lungs.

Cultures. Lungs: Type $\mathrm{C}$ meningococci in almost pure culture recovered from the rigbt lower and the left lower lobes. 
No. 79, C. C. D., Pvt., $2 d$ Bty:, F. A. R.R.

Influenza; bronchopneumonia.

Autopsy. Extensive bronchopneumonia involving all the lobes of both lungs.

Cullures. Lungs: Type $\mathrm{C}$ meningococci recovered from the left lower lobes.

No 80, E. A. G., Pvt., 6th Bty., F. A. R. R.

Influensa; bronchopneumonia.

Autopsy. Extensive bronchopneumonia involving all lobes of both lungs.

Cultures. Lungs: Type $\mathrm{C}$ meningococci in almost pure culture recovered from left upper and right middle lobes.

No. 81, L. F. T., Pvt., 1st Bty., F. A. R. R.

Infuenza; bronchopneumonia.

September 28, 1918. Blood cultures, Pasteur tube, Type C meningococci in pure culture.

September 29. Died.

Autopsy. Empyema (right). Extensive bronchopneumonia involving all lobes of the right lung and the lower lobe of the left lung.

Cultures. Heart's blood; Gram-negative diplococi recovered. Not agglutinated by serum on hand. Culture lost.

No. 85, J. M. B.; Pvt., 5th Bty., F. A. R. R.

Influenza; bronchopneumonia.

Scptember 28, 1918. Blood culture in Pasteur tube; Type C meningococci in pure culture.

September 30, 1918. Died.

Autopsy. Extensive bronchopneumonia involving all the lobes of both the lungs.

Cultures. Heart's blood and upper lobe of right lung; Type C meningococci recovered.

One case admitted as "influenza" after two days in hospital turned out to be epidemic cerebrospinal meningitis.

No. 93, M. C., Pvt., F. A. R. R.

Influenza.

September 30, 1918. Sick about twelve hours. 'Temperature, $99^{\circ}$ F. Blood culture in Pasteur tubes; Type A meningococci in pure culture.

October 2. Meningeal symptoms.

Lumbar Puncture. Cloudy fluid showing polynuclear leukocytes and a few Gram-negative diplococci.

Cullure. Type A meningococci.

Diagnosis. Epidemic cerebrospinal meningitis (Type A).

October 2. Blood culture in Pasteur tube; no growth.

Cases Studied in U. S. A. Base Hospital No. 6. The cases of influenza studied at U. S. A. Base Hospital No. 6 were from many camps: and organizations, some directly from: transports arriving 
from the :United States: At this time other respiratory infections, evidently not true infiuenza, began to appear among troops. Numerous blood cultures were taken in many different ways; in none were Gram-negative diplococci obtained.

In a series of fifteen autopsies on patients dying of bronchopneumonia following influenza, from the lungs of only three were meningococci isolated. . These three strains isolated were all Type 9. One of these strains was obtained from a case taken from a transport arriving from the United States.

Protocols. 'No. 5, J. C., Cpl., 351 Supply Co., Q. M. C.

Influenza; bronchopneumonia. Off transport from United States. Influenza epidemic on board. . Died.

Autopsy. Empyema (right). Acute fibrinous pericarditis. Extensive bronchopneumonia involving all the lobes of both lungs.

Cultures: : Lungs: Type B meningococcus recovered.

No. 11, J. D., Pvt., 321, F. A:

Influenza; bronchopneumonia.

October 22, 1918. Died.

Autopsy. Extensive bronchopneumonia involving all the lobes of both lungs.

Cultures. Lungs: Type B meningococcus recovered.

No. 1, C. T., San. Dept., Det. 343, Infantry.

Influenza; bronchopneumonia.

October 20, 1918. Died.

Autopsy. Empyema (right).' Acute purulcnt pericarditis. Extensive bronchopneumonia involving all lobes of both lungs.

Cultures. Lungs: Type B meningococcus recovered.

Other Types of Bacteria Encountered. . In the LeCourneau epidemic eight blood Gram-negative cultures taken from patients with bronchopneumonia showed Gram-negative micrococci of the Streptocococcus-pneumococcus group. In all, cultures taken from pneumonic lungs of the. Streptococcus-pneumococcus group, in varying numbers, were found. Twelve out of twenty-four cultures taken from the heart's blood of crses dying of pneumonia also showed members of this group: No attempt. was made to classify these Gram-positive micrococci; some were green producers, some hemolytic and a few, growing as large, mucoid, green colonies and showing a definite capsule in stained specimens, were probably either pneumococci Type 3-or Streptococcus mucosus. During the study of this epidemic three cases of epidemic cerebrospinal meningitis occurred in the camp hospital. The meningococci cultured from the spinal fluid of all these three cases were agglutinated by Type A serum, not by Type B, C or normal horse serum. From one of these cases a blood culture, positive for Type A meningococcus was obtained two days before the patient developed meningeal symptoms: 
In a series of fifteen autopsies at Base Hospital No. 6, hemolytic streptococci, Streptococcus viridans, pneumococcus Type 3 and pneumococcus Type 4 were recovered from the heart's blood and lungs in the different cases. In two instances Pfeiffer's bacillus was recovered from the lungs.

During the epidemic of influenza there was a moderate number of scattered cases of epidemic cerebrospinal meningitis. Meningococci recovered from the spinal fluids of the majority of these cases were Types $\mathrm{A}$ and $\mathrm{B}$; in only three instances were Type $\mathrm{C}$ meningococci recovered.

ADDENDun. Since writing this report a small epidemic of "influenza" in "pure", form occurred in Company " $G$," Ammunition Train, stationed at St. Andre de Cubzac, an organization which had escaped the disease during the main epidemic. Forty cases occurred in this organization December 1 and 2. December 4, nasopharyngeal cultures were taken from 29 of these 40 cases. From 16, or 55 per cent., of the cultures taken, Gram-negative micrococci, morphologically and culturally, were isolated. All these microörganisms fermented dextrose and maltose and not saccharosc. Fourteen of the sixteen strains isolated were agglutinated in dilutions of 1 to 50 and 1 to 100 at $52^{\circ} \mathrm{C}$. for eightecn hours by polyvalent New York City Department of Health antimeningitis serum and not by normal horse serum in dilution of 1 to 25 . Two strains could not be agglutinated by any antimeningococcus serum on hand. Of the agglutinable strains it was possible to type only two with the serum on hand. These two strains were Type B. Repeated attempts at typing the other strains were made, both at $37^{\circ} \mathrm{C}$., with sevcral different lots of sera, but with consistently negative results.

- In connection with the I. Courneau epidemic a high percentage of patients in this later epidemic barboring meningococci in their throats is of interest, and by typing it was hoped possibly to throw more light on the etiology of the disease.

From the throats of three of these twenty-nine patients, Pfeiffer's bacillus was recovered.

Discussion. From the above findings no definite conclusions can be drawn. The findings in the Le Courneau epidemic, takcn alone, might suggest that Type $\mathrm{C}$ meningococcus bears an important etiological relation to the cases of disease from which it was obtained. However, study of cases in different localities where a similar or identical disease was prevalent does not bear this out, as Type B meningococcus was recovered at autopsy from the lungs of a certain number of those cases. On the other hand it can be stated that the meningococcus group of bacteria is associated in some way with certain cases of the so-called influenza which prevailed among the American Expeditionary Force in France.

The foregoing studies were made during the early autumn of 
1918. It is therefore interesting to see that a somewhat similar study carried out at Southampton by:Captain William Fletcher! yielded similar results.: : Captain Fletcher. states that between September 25 and November 23, 1918, cultures were made after death from the lungs and heart's blood of 36 American' soldiers who had died of postinfluenzal bronchopneumonia. The illness of these men arose a week or ten days before on transports crossing the Atlantic. In 11 of the 36 cases the lungs and in 3 instances tbe heart's blood yielded numerous colonies of Gram-negative cocci. Subcultures of these cocci from 7 cases were examined serologically. six were found to be Type II and 1 Type Imeningococcus (Gordon's classification). The other four strains had been lost. In all the 11 cases; Bacillus influenzes was present in the lungs in association with the meningococcus. In 2 instances pneumococci, in 1 streptococci and in still another streptococci and pneumococci were found. A British soldier suffering from bronchitis at about the same time yielded from his frothy and purulent sputum a Type IV meningococcus.

Comparing the results obtained in France by us and in England by Fletcher the following -may be stated: Nicolle's Type B meningococcus corresponds with Gordon's Type II while Nicolle's Type $C$ has no counterpart among Gordon's types.' Hence, using Gordon's classification, the type meningococci obtained from tbe heart's blood and lungs of cases of influenza occurring from late September to late November, especially in the American Expeditionary Force, have been chiefly Type II.

The postinfluenzal bronchopneumonias have shown a varied bacteriological flora on culture. This flora, moreover, conforms with the large number of pathogenic bacteria, the Bacillus influenzse possibly excepted, occurring in tbe upper respiratory tract. It is now known that meningococcus is a not infrequent inhabitant of the nasopbarynx. Moreover, during the autumn epidemic meningitis was not infrequent among the American Expeditionary Force. Hence carriers of meningococci were also common. It is therefore probable that the occurrence of meningococci in the lungs and heart closely parallels the occurrence of streptococci and pneumococci, only that the latter are more infrequent. All are to be viewed as secondary invaders and concomitants and not primary microbic causes of influenza. But as meningococci are hardly regarded as being concerned in any manner with bronchopneumonia, it appears proper that the findings we bave made should be recorded.

' Lascet, Janurary 18, 1019, p. 104: 